\title{
Verification of Contact Stress with Surrogate Duality
}

\author{
Zhaocheng Xuan* \\ Department of Computer Science, Tianjin University of Technology and Education, Tianjin 300222, China \\ *Corresponding author
}

\begin{abstract}
We present a method for verifying the contact stress of elastic bodies. An explicit formulation of the total contact force, a fraction function with the numerator as a linear function and the denominator as a quadratic convex function, is derived by using the surrogate model of quadratic optimization for the contact problem. Then a bound formulation is obtained for the sum of the nodal contact forces, which is an explicit formulation of matrices of the finite element model, derived by maximizing the fraction function under the constraint that the sum of the normalized nodal contact forces is one. The bound is solved with the problem dimensions being only the number of contact nodes or node pairs, which are much smaller than the dimension for the original problem. Next, a scheme for constructing an upper bound on the contact stress is proposed that uses the bound on the sum of the nodal contact forces obtained on a fine finite element mesh and the nodal contact forces obtained on a coarse finite element mesh. Finally, the proposed method is verified through an example to demonstrate its feasibility and robustness.
\end{abstract}

Keywords-contact stress; bound; surrogate; verification; finite elements

\section{INTRODUCTION}

Based on the principle of minimum potential energy, an elastic contact problem is essentially an optimization problem with the contact condition as a constraint. In the Karush-KuhnTucker (KKT) conditions for these optimization problems, the Lagrange multipliers that represent the nodal forces are in an unbounded positive space. It is fortunate that when aggregating the constraints with a so-called surrogate constraint $[1,2]$, we obtain an explicit formulation of the sum of the nodal contact forces, and the variable - the normalized nodal contact force - is only constrained in a bounded simplex. Therefore, it is possible to find an upper bound on the sum of nodal contact forces, and when this is done, we can obtain a bound on the contact stress with the information provided by the normalized nodal contact forces solved with a coarse mesh.

For construction of the bounded feasible region, we have to solve an optimization problem with an objective being a fraction function. We first prove that the objective is pseudo concave in a neighborhood of the optimum, and then, with a suitable initial solution, optimization methods can be used to solve the fractional programming problem to obtain the optimum value. In this paper, we construct a bound that is explicitly formulated in terms of matrices regarding the finite element model, by maximizing the fraction function with a larger constraint field.

\section{A Surrogate Optimization Model of the Contact PROBLEM}

A surrogate duality for optimization was proposed by [1,2] for solving a zero-one programming problem. The authors used a single constraint that is a positive linear combination of the original constraints in place of those constraints to form a surrogate optimization problem, and then provided a surrogate duality problem for the primal optimization problem. Analogous to Glover's idea for solving a zero-one programming problem, the surrogate constraint problem for the primal problem has the form Units

$$
\min \left\{\pi(u): \lambda^{\mathrm{T}}(\mathrm{Au}-\mathrm{g}) \leq 0\right\}
$$

where $\lambda=\left\{\lambda_{1}, \lambda_{2}, \cdots, \lambda_{m}\right\}^{\mathrm{T}}$ is a vector composed of the nonnegative weights, termed surrogate multipliers, which may be normalized without loss of generality by requiring that

$$
\mathrm{e}^{\mathrm{T}} \lambda=1
$$

We use $\Delta=\left\{\lambda \in \mathfrak{R}^{\mathrm{m}} \mid \mathrm{e}^{\mathrm{T}} \lambda=1, \lambda \geq 0\right\}$, termed as the canonical or simplex form, to represent the constraints, where e is a vector composed of ones. A surrogate dual objective can be defined as

$$
s(\lambda)=\min \left\{\pi(u): \lambda^{T}(A u-g) \leq 0\right\}
$$

which is the minimum value of $\pi(u)$ at the solution point of problem (1) for fixed $\lambda \geq 0$, which of course satisfies the normality condition (2). Then the surrogate dual problem is:

$$
\max \{s(\lambda): \lambda \in \Delta\}
$$

It can be seen that the constraint of problem (1) is very simple, and for a continuous smooth convex optimization problem, there is no gap between the primal problem (1) and the dual problem (4) [2].

For surrogate constraint problem (3), the Lagrangian function is

$\mathrm{L}(\mathrm{u}, \lambda, \alpha, \beta, \gamma)=\pi(\mathrm{u})+\alpha \lambda^{\mathrm{T}}(\mathrm{Au}-\mathrm{g})+\beta\left(\mathrm{e}^{\mathrm{T}} \lambda-1\right)+\gamma^{\mathrm{T}} \lambda(5)$

where $\alpha, \beta$, and $\gamma$ are Lagrange multipliers. The saddle point conditions with respect to $u$ and $\lambda$ are respectively

$$
\begin{gathered}
\nabla_{\mathrm{u}} \mathrm{L}(\mathrm{u}, \lambda, \alpha, \beta, \gamma)=\mathrm{Ku}-\mathrm{p}+\alpha \mathrm{A}^{\mathrm{T}} \lambda=0 \\
\nabla_{\lambda} \mathrm{L}(\mathrm{u}, \lambda, \alpha, \beta, \gamma)=\alpha(\mathrm{Au}-\mathrm{g})+\beta \mathrm{e}+\gamma=0
\end{gathered}
$$

and the complementary conditions are as follows:

$$
\alpha \lambda^{\mathrm{T}}(\mathrm{Au}-\mathrm{g})=0
$$




$$
\begin{gathered}
\beta\left(\mathrm{e}^{\mathrm{T}} \lambda-1\right)=0 \\
\gamma^{\mathrm{T}} \lambda=0
\end{gathered}
$$

It is interesting that if we dot multiply $\lambda$ with both sides of equation (4) and with equations (7), (8), and (9), we find that $\beta$ is zero. That means that the constraint $\mathrm{e}^{\mathrm{T}} \lambda-1$ does not influence the optimum solution for primal problem (1). This is an alternative way to prove there is no gap between problem (1) and problem (3), and therefore no gap between problem (1) and problem (4).

Now we derive the relationships of $\mathrm{u}, \lambda$, and $\alpha$ from stationary condition (6) and complementary condition (7). Using condition (6), we obtain $\mathrm{u}$ with respect to $\lambda$ and $\alpha$ :

$$
\mathrm{u}=\mathrm{K}^{-1}\left(\mathrm{p}-\alpha \mathrm{A}^{\mathrm{T}} \lambda\right)
$$

Turning to condition (7), there are two possibilities for $\alpha$ : either $\alpha=0$ or $\alpha \neq 0$. If $\alpha=0$, then it might be that $\lambda^{\mathrm{T}}(\mathrm{Au}-$ g) $\leq 0$, which means that the unconstrained minimum of the surrogate constraint problem (3)

$$
\mathrm{u}^{0}=\arg \min \pi(\mathrm{u})
$$

is the solution, and it is the solution of the elastic problem without considering contact. If $\alpha \neq 0$, then it must be the case that

$$
\lambda^{\mathrm{T}}(\mathrm{Au}-\mathrm{g})=0
$$

Before finding the equation for $\alpha$, let us give a property of the kinematic transformation matrix A with the following lemma. rank.

Lemma The kinematic transformation matrix $\mathrm{A}$ is full row

Proof: For simplicity, we only use two dimensional problems to prove the lemma. Referring to Figure 1, the nonpenetration condition for node pair $\mathrm{i}-\mathrm{i}^{\prime}$ is

$$
-u_{i}^{\mathrm{x}} \cos \theta-u_{i}^{\mathrm{y}} \sin \theta+u_{i^{\prime}}^{\mathrm{x}} \cos \theta+u_{i^{\prime}}^{\mathrm{y}} \sin \theta \leq \mathrm{g}_{\mathrm{i}}
$$

and therefore the row of kinematics transformation matrix $\mathrm{A}$ corresponding to the above constraint is

$$
0 \cdots 0 \overbrace{-\cos \theta}^{2 \mathrm{i}-1} \overbrace{-\sin \theta}^{2 \mathrm{i}} 0 \cdots 0 \overbrace{\cos \theta}^{2 \mathrm{i}^{\prime}-1} \overbrace{\sin \theta}^{2 \mathrm{i}^{\prime}} 0 \cdots 0
$$

There are nonzero terms at columns $2 \mathrm{i}-1,2 \mathrm{i}, 2 \mathrm{i}^{\prime}-1$, and $2 \mathrm{i}^{\prime}$ for this row vector, while for all other row vectors of matrix $\mathrm{A}$, the terms at columns $2 \mathrm{i}-1,2 \mathrm{i}, 2 \mathrm{i}^{\prime}-1$, and $2 \mathrm{i}^{\prime}$ are all zeros. This is true for every row of matrix $A$, which leads to the conclusion that matrix $A$ is full row rank.

Combining (12) with (11), we have

$$
\alpha=\frac{\mathrm{d}^{\mathrm{T}} \lambda}{\lambda^{\mathrm{T}} \mathrm{B} \lambda}
$$

where $d=A K^{-1} p-g$ and $B=A K^{-1} A^{T}$. Matrix $K^{-1}$ is positive definite and $A$ is full row rank, so $\lambda^{T} A K^{-1} A^{T} \lambda>0$ for all $\lambda \in \Delta$

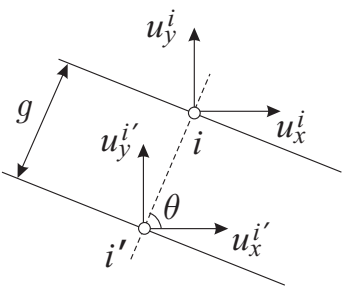

FIGURE I. AN ILLUSTRATION OF A CONTACT NODE PAIR

The primal problem is the principle of minimum total potential energy under the constraint of contact displacement, and its Lagrangian function is

$$
L(u, \xi)=\pi(u)+\xi^{T}(A u-g)
$$

where $\xi$ is the vector of Lagrange multipliers; the physical meaning of $\xi$ is the nodal contact forces on the potential contact nodes. Comparing the two Lagrangian functions (18) and (30), we can see that the product of the Lagrange multiplier $\alpha$ and surrogate multiplier $\lambda$ is actually the Lagrange multiplier $\xi$, that is,

$$
\alpha \lambda=\xi
$$

and as $\mathrm{e}^{\mathrm{T}} \lambda=1$, we obtain

$$
\alpha=\mathrm{e}^{\mathrm{T}} \xi
$$

and

$$
\lambda=\frac{\xi}{\mathrm{e}^{\mathrm{T} \xi}}
$$

Therefore, we have the following theorem.

Theorem 1 The Lagrange multiplier $\alpha$ of the surrogate dual problem is the sum of the nodal contact forces, and the surrogate multipliers $\lambda$ are the normalized nodal contact forces

From equation (29), we can see that $\alpha$ is a fractional function with a linear function of $\lambda$ as the numerator and a quadratic function of $\lambda$ as the denominator. As stated above, matrix B is positive definite, so the denominator is always greater than zero. For the numerator $\mathrm{d}^{\mathrm{T}} \lambda$, as $\mathrm{d}=\mathrm{AK}^{-1} \mathrm{p}-\mathrm{g}=$ $\mathrm{Au}^{0}-\mathrm{g}$, there will be three cases of the contact state. The first is that the unconstrained minimum $\mathrm{u}^{0}$ satisfies all constraints $\mathrm{Au}-\mathrm{g} \leq 0$, that is, $\mathrm{d} \leq 0$. In this case there is no contact, so we will ignore this case. The second is that $d>0$, which means that the unconstrained minimum violates all constraints. The third is that some components of $d$ are less than or equal to zero, and the rest are greater than zero. This third case is the general case, and as defining fewer potential contact nodes than the actual number of contact nodes will lead to incorrect solutions, we choose a number of potential contact nodes that is greater than the actual number to guarantee the correctness of the solution. 
Let us use $\lambda^{*}$ to denote the solution of the KKT conditions; it is the vector of normalized nodal contact forces computed by the finite element method. As $\alpha$ is a function of $\lambda$, we can also use $\alpha(\lambda)$ to express $\alpha$ in equation (29), and because $\lambda^{*} \in \Delta$, it follows that is an upper bound on the sum of nodal contact forces, that is,

$$
\begin{gathered}
\alpha^{*}=\arg \max \{\alpha(\lambda): \lambda \in \Delta\} \\
\frac{\mathrm{d}^{\mathrm{T}} \lambda^{*}}{\lambda^{* \mathrm{~T}} \mathrm{~B} \lambda^{*}} \leq \alpha^{*}
\end{gathered}
$$

Now let us present some properties of $\alpha(\lambda)$.

Theorem 2 There are no stationary points for $\alpha(\lambda)$ in $\Delta$.

Proof: The derivative of $\alpha(\lambda)$ is

$$
\nabla_{\lambda} \alpha(\lambda)=\frac{\mathrm{d} \cdot \lambda^{\mathrm{T}} \mathrm{B} \lambda-2 \mathrm{~d}^{\mathrm{T}} \lambda \cdot \mathrm{B} \lambda}{\left(\lambda^{\mathrm{T}} \mathrm{B} \lambda\right)^{2}}
$$

If we can show there is no $\lambda$ in $\Delta$ to make the numerator of $\alpha(\lambda)$ vanish, then we will have the proof of the lemma. Suppose that $\alpha(\lambda)$ is equal to zero, that is, the numerator is zero: $d \cdot m \lambda^{T} B \lambda-2 d^{T} \lambda \cdot B \lambda=0$. Then, dot-multiplying both sides with $\lambda$, we obtain $\mathrm{d}^{\mathrm{T}} \lambda=0$. This cannot be the case, because $\mathrm{d}^{\mathrm{T}} \lambda=0$ means the unconstrained minimum solution satisfies (does not violate) the constraints, so no contact occurs, but we have already eliminated this case. Therefore, $\nabla_{\lambda} \alpha(\lambda)$ cannot be zero for $\lambda$ in $\Delta$, and the optimal solution must then be on the boundary of $\Delta$.

From the viewpoint of the geometry of $\Delta$, when one component of $\lambda$ is zero, $\lambda$ is on the boundary of $\Delta$. From Lemma 2 and Lemma 3, we know that the maximum $\alpha^{*}$ should be on the boundary of $\Delta$, and the solution $\lambda^{*}$ is also on the boundary of $\Delta$, because we set the number of potential contact nodes to be greater than the actual number of contact nodes. So there must be components of $\lambda^{*}$ that are zeros.

\section{METHODS FOR SOLVING THE UPPER BOUNDS}

One possible method for solving the fractional programming problem (34) is through simplicity optimization by subdivision of the simplex and using the branch and bound algorithm to find the maximum $[3,4,5]$. Now let us construct another bound. We introduce two optimization problems:

$$
s(\bar{\eta})=\max \left\{d^{T} \lambda-\bar{\eta} \lambda^{T} B \lambda: e^{T} \lambda=1\right\}
$$

and

$$
\bar{\alpha}=\arg \max \left\{\alpha(\lambda): \mathrm{e}^{\mathrm{T}} \lambda=1\right\}
$$

These two problems are equivalent according to the properties listed above, and the feasible region is larger than that of problems (34) and (45). Thus we have

$$
\bar{\eta}=\bar{\alpha} \geq \alpha^{*}
$$

Therefore, if we can solve $\bar{\eta}$, then an upper bound on $\alpha^{*}$ has been found, and it is surely an upper bound on the sum of the nodal contact forces. Let us first write out the Lagrangian function for problem (50) as

$$
L(\lambda, \gamma)=d^{T} \lambda-\bar{\eta} \lambda^{T} B \lambda+\gamma\left(e^{T} \lambda-1\right)
$$

where $\gamma$ is the Lagrange multiplier. The stationary condition with respect to $\lambda$ is

$$
d-2 \bar{\eta} B \lambda+\gamma e=0
$$

so we have

$$
\lambda=\frac{1}{2 \bar{\eta}} B^{-1}(d+\gamma e)
$$

Substituting this into the constraint condition $\mathrm{e}^{\mathrm{T}} \lambda=1$, we obtain

$$
\gamma=\frac{1}{\mathrm{e}^{\mathrm{T}} \mathrm{TB}^{-1} \mathrm{e}}\left(\mathrm{e}^{\mathrm{T}} \mathrm{B}^{-1} \mathrm{~d}-2 \bar{\eta}\right)
$$

and substituting this into (55), $\lambda$ is then expressed as a function of $\bar{\eta}$ :

$$
\lambda=\frac{1}{2 \bar{\eta}}\left(\mathrm{B}^{-1} \mathrm{~d}-\frac{\mathrm{e}^{\mathrm{T}} \mathrm{B}^{-1} \mathrm{~d}}{\mathrm{e}^{\mathrm{T}} \mathrm{B}^{-1} \mathrm{e}} \mathrm{B}^{-1} \mathrm{e}\right)+\frac{1}{\mathrm{e}^{\mathrm{T}} \mathrm{B}^{-1} \mathrm{e}} \mathrm{B}^{-1} \mathrm{e}
$$

This is a general formulation for $\lambda$ that satisfies the constraint $\mathrm{e}^{\mathrm{T}} \lambda=1$. Through adjusting $\bar{\eta}$ we can make each component of $\lambda$ greater than or equal to zero, that is, we can make it satisfy the constraint $\Delta$ - but this may conflict with the condition that the objective should be zero, that is, $\mathrm{d}^{\mathrm{T}} \lambda-\bar{\eta} \lambda^{\mathrm{T}} \mathrm{B} \lambda=0$; so in this case we cannot guarantee that $\lambda \in \Delta$, but we can guarantee that $\bar{\eta}$ is an upper bound on $\alpha^{*}$ if $\bar{\eta}$ satisfies $d^{\mathrm{T}} \lambda-\bar{\eta} \lambda^{\mathrm{T}} \mathrm{B} \lambda=0$.

Therefore, substituting $\lambda$ into the objective, we make it zero, and with some manipulation, we obtain the following equation for $\bar{\eta}$ :

$$
\bar{\eta}^{2}-e^{T} B^{-1} d \bar{\eta}+\frac{1}{4}\left(\left(e^{T} B^{-1} d\right)^{2}-e^{T} B^{-1} e \cdot d^{T} B^{-1} d\right)=0(32)
$$

Solving the equation, we have

$$
\bar{\eta}=\frac{1}{2}\left(e^{T} B^{-1} d \pm \sqrt{e^{T} B^{-1} e \cdot d^{T} B^{-1} d}\right)
$$

According to the Cauchy-Schwarz inequality, $\mathrm{e}^{\mathrm{T}} \mathrm{B}^{-1} \mathrm{~d} \leq$ $\sqrt{\mathrm{e}^{\mathrm{T}} \mathrm{B}^{-1} \mathrm{e} \cdot \mathrm{d}^{\mathrm{T}} \mathrm{B}^{-1} \mathrm{~d}}$, so the minus sign in the above equation can be omitted because $\bar{\eta}$ should be greater than zero, giving us

$$
\bar{\eta}=\frac{1}{2}\left(e^{T} B^{-1} d+\sqrt{e^{T} B^{-1} e \cdot d^{T} B^{-1} d}\right)
$$

This is referred to as Bound 2 in the illustration examples that follow.

Once the bound on the sum of the nodal contact forces has been produced, it can be used to provide an upper bound on the maximal contact stress on the fine finite element meshes. 
One approach for doing this is to use the obtained bound on the fine meshes, and $\lambda$ on the coarse mesh. $\lambda$ represents the normalized contact forces, giving the distribution of the contact forces on the coarse mesh. We then use BOUND for the total contact forces, and multiply it by $\lambda$ to find the maximal contact force from all contact nodes of the coarse mesh, that is,

$$
\max _{\mathrm{i}} \frac{\lambda_{\mathrm{i}}}{\mathrm{s}_{\mathrm{i}}} \text { BOUND }
$$

where $s_{i}$ is the area on which $\lambda_{i}$ acts. We can certainly use $\lambda$ on the fine meshes to calculate the above solution, but this would be computationally expensive, so $\lambda$ with the inexpensive computation on the coarse mesh is enough for providing the bounds on the maximal contact stress. Since $\lambda$ is only a normalized solution of contact forces, it only represents a distribution of the contact forces.

\section{ILLUSTRATION EXAMPLES}

In this section, we examine some examples to verify the correctness and efficiency of the methods proposed in the previous sections for calculating the two bounds. The examples involve the nodal forces produced from the contact between an elastic body and a rigid foundation and the contact between two elastic bodies, and one example also involves friction between an elastic body and a rigid foundation.

Example: An elastic slab in a state of plane strain is fixed at two ends and pressed on a foundation as shown in Figure 3. The Young's modulus of the elastic body is $\mathrm{E}=2900 \mathrm{~N} / \mathrm{cm}^{2}$, the Poisson's ratio is $v=0.4$, and the pressure is $p=$ $10 \mathrm{~N} / \mathrm{cm}^{2}$. The dimensions of the structure in centimeters are shown in the figure. There is a gap between the elastic body and the foundation. In this example, we will consider two different gaps in two cases of the foundation to compute the bounds: one is a rigid foundation and the other is an elastic foundation. Due to symmetry, only the left half of the structure is used to construct the finite element model.

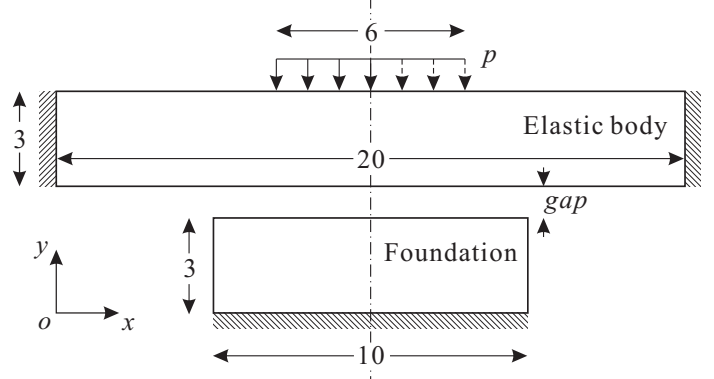

FIGURE II. AN ELASTIC SLAB COMING INTO CONTACT WITH A FOUNDATION

We set the length of the potential contact edge to 5 , half the length of the foundation. Figure 4 shows the finite element mesh and 11 potential contact node pairs arranged on the potential contact edges. For illustrating the bounds on the sum of nodal contact forces, we use different meshes with smaller mesh sizes than the coarse mesh shown in Figure 3, namely, $\mathrm{H} / 2, \mathrm{H} / 4, \mathrm{H} / 8$, and $\mathrm{H} / 16$. Tables 1 and 2 give the results of the two bounds and the sum of the nodal contact forces with respect to the different mesh sizes in the cases of a rigid foundation and an elastic foundation, respectively, with the gap being 0.1 .

\section{CONCLUSIONS}

In this paper, we provide a practical upper bound on the contact stress in contact problems. The bound is explicitly formulated, obtained by maximizing the objective of nodal contact forces with a larger feasible region An example is provided to show that the method can provide an upper bound on the contact stress calculated by a finite element method on very fine meshes. More detailed content can be found in one author's paper to be published in Computational Mechanics.

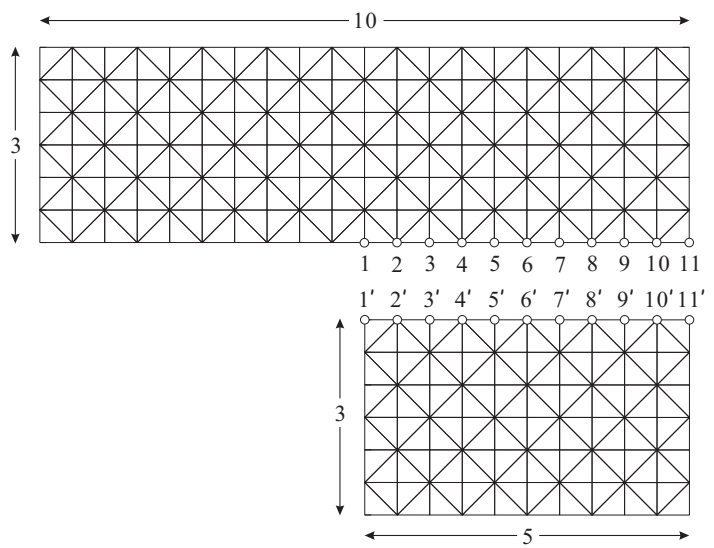

FIGURE III. THE COARSE MESH WITH MESH SIZE H AND THE POTENTIAL CONTACT NODE PAIRS

TABLE I. THE CASE OF A RIGID FOUNDATION WITH GAP $=0.1$

\begin{tabular}{|c|c|c|c|c|c|}
\hline Mesh size & $\mathrm{H}$ & $\mathrm{H} / 2$ & $\mathrm{H} / 4$ & $\mathrm{H} / 8$ & $\mathrm{H} / 16$ \\
\hline Bound & 21.8222 & 22.6050 & 22.8171 & 22.8766 & 22.8939 \\
\hline$\alpha$ & 19.5280 & 20.0489 & 20.1542 & 20.2415 & 20.2608 \\
\hline
\end{tabular}

TABLE II. THE CASE OF AN ELASTIC FOUNDATION WITH GAP $=0.1$

\begin{tabular}{|c|c|c|c|c|c|}
\hline Mesh size & $\mathrm{H}$ & $\mathrm{H} / 2$ & $\mathrm{H} / 4$ & $\mathrm{H} / 8$ & $\mathrm{H} / 16$ \\
\hline Bound & 21.1990 & 21.9368 & 22.1475 & 22.2122 & 22.2337 \\
\hline$\alpha$ & 19.3392 & 19.9082 & 20.0769 & 20.1308 & 20.1493 \\
\hline
\end{tabular}

\section{ACKNOWLEDGEMENT}

This work is supported by the National Natural Science Foundation of China under Grant number 11172209.

\section{REFERENCES}

[1] F. Glover, Surrogate constraints, Operations Research, 16: 741-749, 1968.

[2] H.J. Greenberg, W.P. Pierskalla, Surrogate mathematical programming, Operations Research, 18: 924-939, 1970.

[3] R. Paulavičius, J. Žilinskas, Simplicial Global Optimization, Springer, 2014.

[4] H. Edelsbrunner, D.R. Grayson, Edgewise subdivision of a simplex, Proceeding SCG '99 Proceedings of the Fifteenth Annual Symposium on Computational Geometry, 24-30.

[5] E.N. Gonçalves, R.M. Palhares, R.H.C. Takahashi, R.C. Mesquita, Algorithm 860: SimpleS - an extension of Freudenthal's simplex subdivision, ACM Transactions on Mathematical Software, 32: 609-621, 2006. 